\title{
Tuning the Thickness of a Biomembrane by Stapling Diamidophospholipids with Bolalipids
}

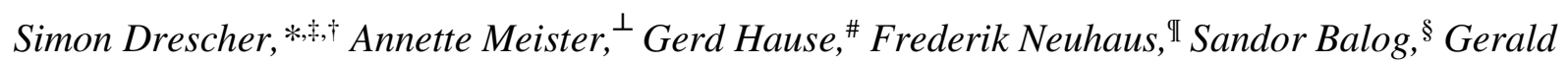
Brezesinski, I and Andreas Zumbuehl ${ }^{*, \mathbb{I}, \Delta}$

¥ Institute of Pharmacy—Biophysical Pharmacy, Martin Luther University (MLU) Halle-Wittenberg, Wolfgang-Langenbeck-Str. 4, 06120 Halle (Saale), Germany

$\dagger$ Phospholipid Research Center, Im Neuenheimer Feld 515, 69120 Heidelberg, Germany

${ }^{\perp}$ ZIK HALOmem and Institute of Biochemistry and Biotechnology, Charles Tanford Protein

Center, MLU Halle-Wittenberg, Kurt-Mothes-Str. 3a, 06120 Halle (Saale), Germany

\# Biocenter, MLU Halle-Wittenberg, Weinbergweg 22, 06120 Halle (Saale), Germany

II National Centre of Competence in Research in Chemical Biology, 1211 Geneva, Switzerland

$\S$ Adolphe-Merkle-Institute, University of Fribourg, Chemin des Verdiers 4, 1700 Fribourg, Switzerland

॥ Max Planck Institute of Colloids and Interfaces, Science Park Potsdam-Golm, Am Mühlenberg 1, 14476 Potsdam, Germany

${ }^{\Delta}$ Acthera Therapeutics Ltd. Peter Merian-Str. 45, 4052 Basel, Switzerland

\section{Content:}

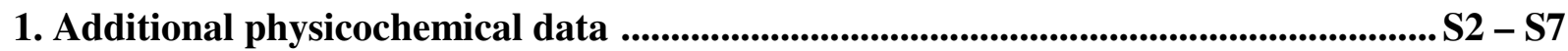

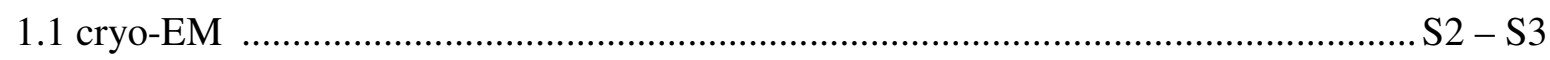

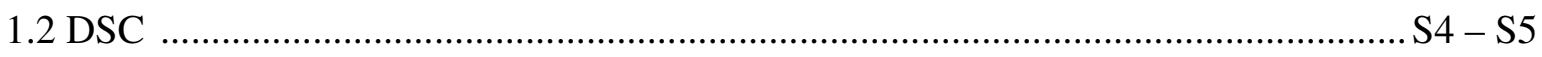

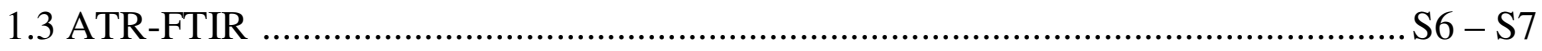




\section{SUPPORTING INFORMATION}

\section{Additional physicochemical data}

\section{1 cryo-EM}

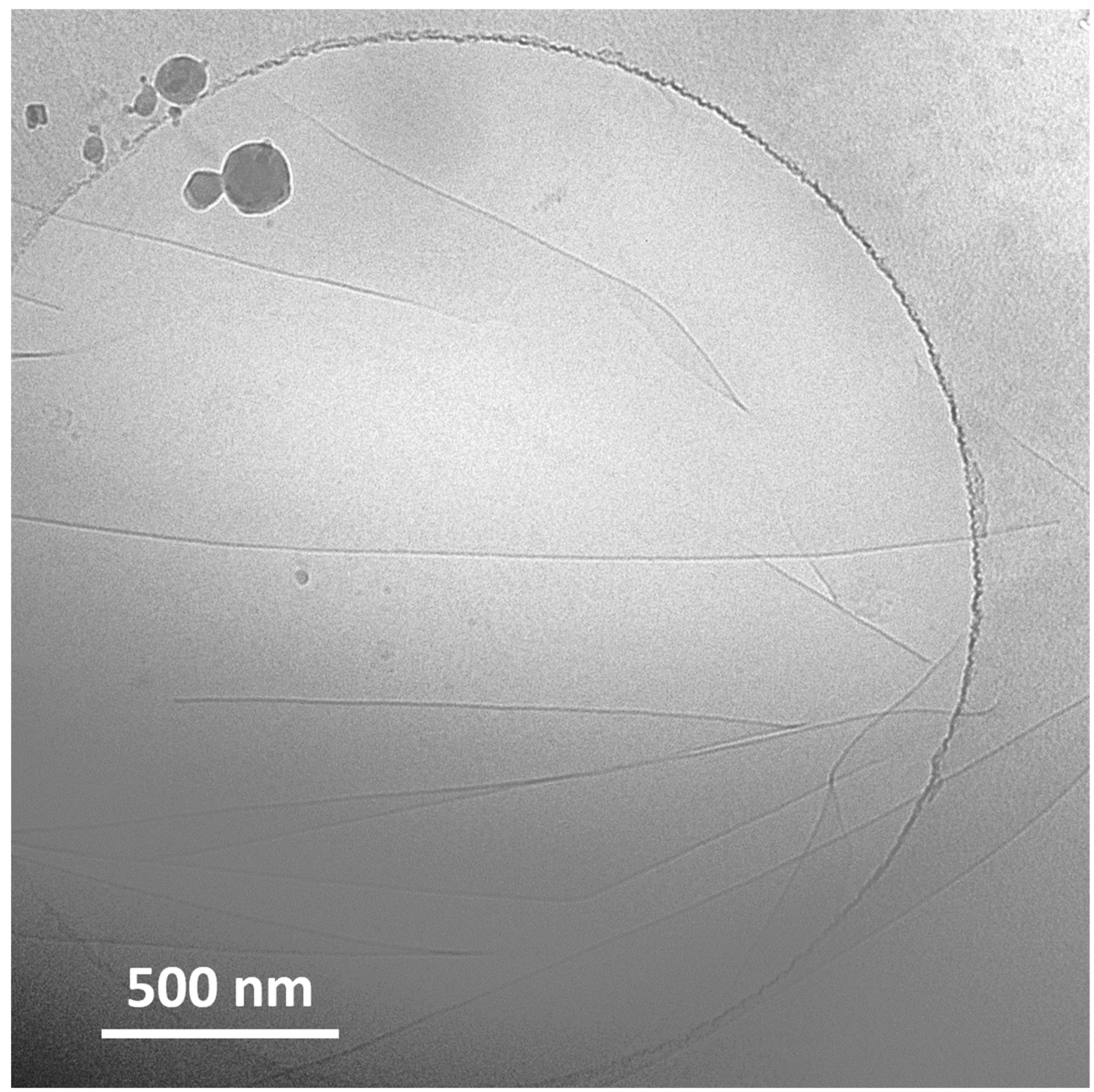

Figure S1. Cryo-EM image of aqueous suspension $(c=2 \mathrm{mM})$ of Pad-PC-Pad mixture with $\mathrm{Me}_{2} \mathrm{PE}-\mathrm{C} 22-\mathrm{Me}_{2} \mathrm{PE}(2 / 1, n / n)$; sample was quenched from $22{ }^{\circ} \mathrm{C}$. 


\section{SUPPORTING INFORMATION}

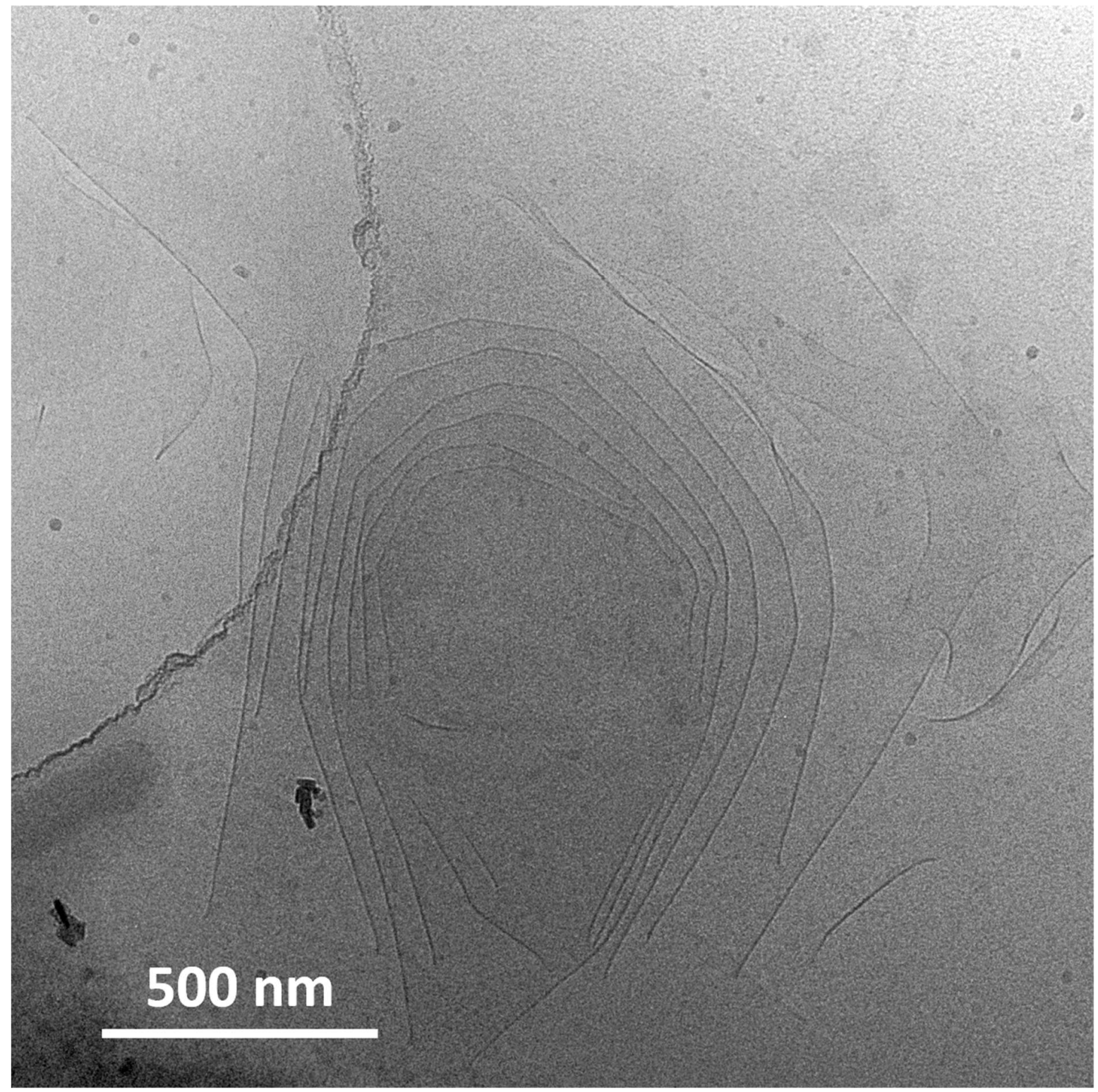

Figure S2. Cryo-EM image of aqueous suspension $(c=2 \mathrm{mM})$ of Pad-PC-Pad mixture with $\mathrm{Me}_{2} \mathrm{PE}-\mathrm{C} 24-\mathrm{Me}_{2} \mathrm{PE}(2 / 1, n / n)$; sample was quenched from $22^{\circ} \mathrm{C}$. 


\section{SUPPORTING INFORMATION}

\subsection{DSC}

\section{$\underline{\mathrm{Pad}-\mathrm{PC}-\mathrm{Pad} / \mathrm{Me}_{2}} \mathrm{PE}_{2} \mathrm{C} 22-\mathrm{Me}_{2} \mathrm{PE}(2 / 1, n / n)$}

Raw data of measurement (PL):

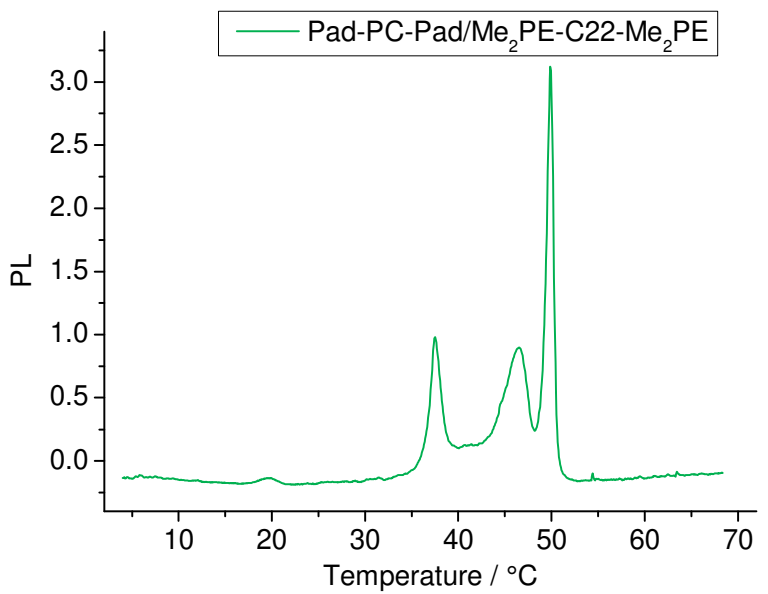

\section{$\underline{\mathrm{Pad}-\mathrm{PC}-\mathrm{Pad} / \mathrm{Me}_{2}} \underline{\mathrm{PE}-\mathrm{C} 24-\mathrm{Me}_{2}} \underline{\mathrm{PE}(2 / 1, n / n)}$}

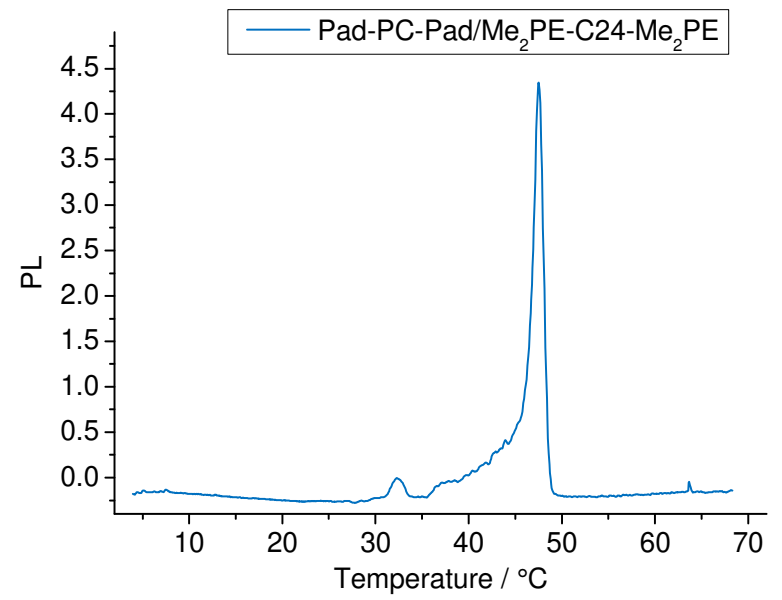

Baseline (BL) phosphate buffer at pH 7.6, $10 \mathrm{mM}$ :

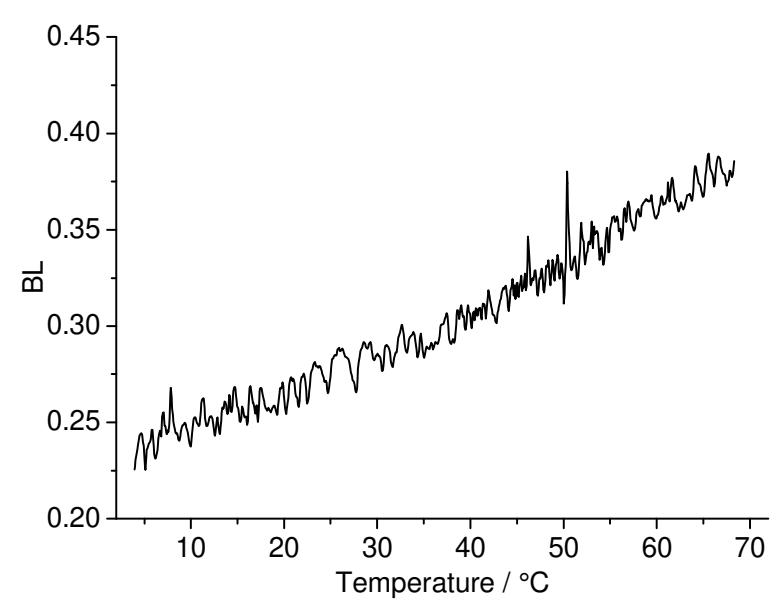

For the calculation of the $C_{\mathrm{p}}$-values, we used the following formula to obtain the DSC curves shown in the manuscript

$$
C_{p}=\left(0.95+\frac{P L-B L}{0.518}\right) \cdot M_{w} \cdot 0.00418
$$

where 0.95 is the instrument constant, 0.518 the volume of the sample cell [mL], $M_{\mathrm{w}}$ the mean molecular weight of the mixture, and 0.00418 the conversion factor from [cal] to [kJ]. The cooling curves were calculated in the same way. 


\section{SUPPORTING INFORMATION}

Table S1. Data taken from DSC measurements of aqueous suspensions of pure lipids and lipid mixtures, respectively. Data analysis was performed using Origin 8.0 software. (FWHM = full width at half maximum)

\begin{tabular}{|c|c|c|c|c|c|c|c|c|c|}
\hline \multirow[b]{2}{*}{ Lipid } & & \multirow[b]{2}{*}{ Peak } & \multirow{2}{*}{$\begin{array}{l}\text { Enthalpy } \\
\left(\Delta H_{p} / \mathrm{kJ} \mathrm{mol}^{-1}\right)\end{array}$} & \multicolumn{3}{|c|}{ Transition $\left({ }^{\circ} \mathrm{C}\right)$} & \multirow{2}{*}{$\begin{array}{l}\text { FWHM } \\
(\mathrm{K})\end{array}$} & \multirow{2}{*}{$\begin{array}{l}\text { Left half } \\
\text { width }(\mathrm{K})\end{array}$} & \multirow{2}{*}{$\begin{array}{l}\text { Right half } \\
\text { width }(K)\end{array}$} \\
\hline & & & & Begin & End & Maximum & & & \\
\hline \multicolumn{10}{|c|}{ Pad-PC-Pad } \\
\hline & heating 1 & & 36.7 & 35.0 & 38.3 & 37.1 & 0.40 & 0.21 & 0.19 \\
\hline & cooling 1 & & -33.3 & 31.6 & 35.4 & 33.0 & 1.05 & 0.50 & 0.55 \\
\hline \multicolumn{10}{|c|}{$\mathrm{Me}_{2} \mathrm{PE}-\mathrm{C} 22-\mathrm{Me}_{2} \mathrm{PE}$} \\
\hline & heating 1 & & 20.5 & 14.6 & 21.6 & 20.1 & 1.56 & 0.99 & 0.56 \\
\hline & cooling 1 & & -20.1 & 9.9 & 20.8 & 18.0 & 1.97 & 1.23 & 0.74 \\
\hline \multicolumn{10}{|c|}{$\mathrm{Me}_{2} \mathrm{PE}-\mathrm{C} 24-\mathrm{Me}_{2} \mathrm{PE}$} \\
\hline & heating 1 & 1 & 15.7 & 28.1 & 31.7 & 30.3 & 1.34 & 0.79 & 0.55 \\
\hline & cooling 1 & 1 & -12.5 & 14.2 & 27.1 & 23.3 & 3.83 & 2.23 & 1.60 \\
\hline \multicolumn{10}{|c|}{ Pad-PC-Pad/Me $/ \mathrm{ME}_{2} \mathrm{PE} 22-\mathrm{Me}_{2} \mathrm{PE}(2 / 1, n / n)$} \\
\hline & heating & 1 & 7.8 & 35.5 & 39.7 & 37.5 & 1.23 & 0.55 & 0.67 \\
\hline & & 2 & 14.9 & 42.2 & 48.2 & 46.6 & 2.82 & 1.75 & 1.06 \\
\hline & & 3 & 19.0 & 48.2 & 51.6 & 49.9 & 0.87 & 0.48 & 0.39 \\
\hline & cooling & 1 & -9.3 & 31.6 & 37.0 & 35.0 & 2.53 & 1.30 & 1.23 \\
\hline & & 2 & -10.7 & 37.1 & 42.2 & 40.0 & 2.18 & 1.18 & 1.00 \\
\hline & & 3 & -15.4 & 42.4 & 47.9 & 45.7 & 1.48 & 0.84 & 0.63 \\
\hline \multicolumn{10}{|c|}{ Pad-PC-Pad/Me 2 PE-C24-Me 2 PE $(2 / 1, n / n)$} \\
\hline & heating & 1 & 59.5 & 35.6 & 49.2 & 47.5 & 1.35 & 0.75 & 0.60 \\
\hline & cooling & 1 & -66.0 & 28.6 & 47.1 & 44.4 & 1.19 & 0.68 & 0.50 \\
\hline
\end{tabular}




\section{SUPPORTING INFORMATION}

\subsection{ATR-FTIR}
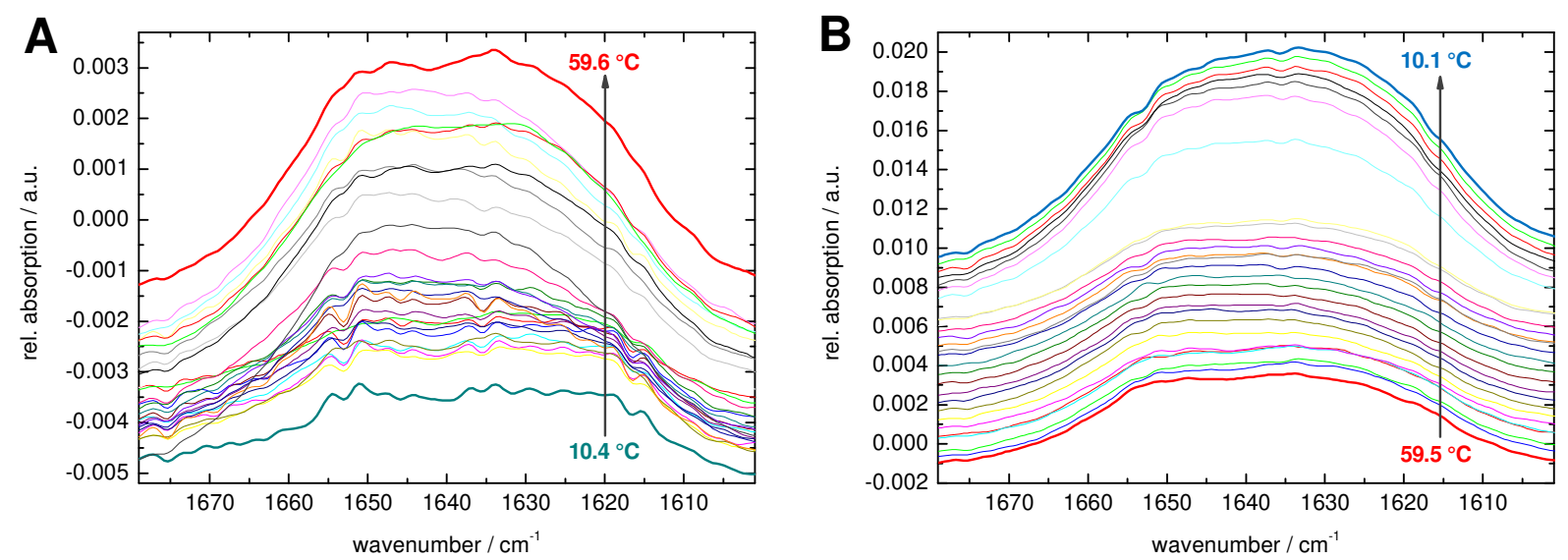

Figure S3. ATR-FTIR measurements of a Pad-PC-Pad/Me ${ }_{2} \mathrm{PE}-\mathrm{C} 22-\mathrm{Me}_{2} \mathrm{PE}$ mixture $(2 / 1, n / n)$ in $\mathrm{D}_{2} \mathrm{O}(c=30 \mathrm{mM})$ presenting the amide I region of the infrared spectra during heating $(\mathrm{A})$ and cooling scans (B). The curves are shifted vertically for clarity.
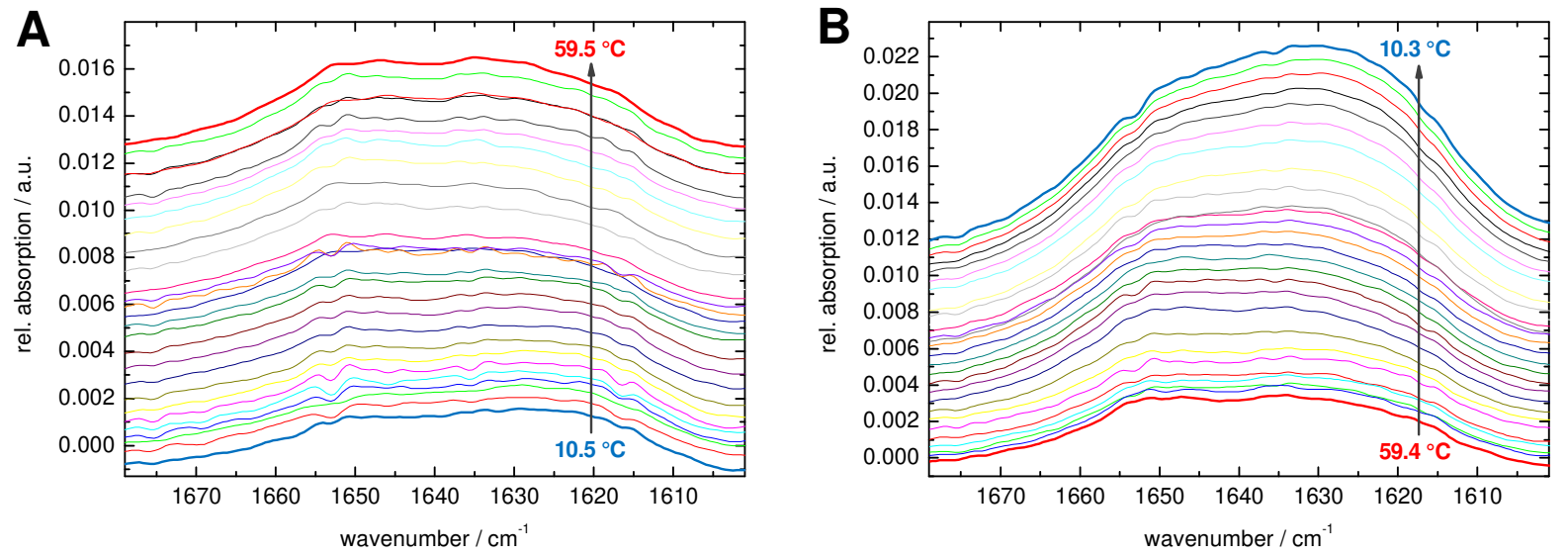

Figure S4. ATR-FTIR measurements of a Pad-PC-Pad/Me $\mathrm{Me}_{2} \mathrm{PE}-\mathrm{C} 24-\mathrm{Me}_{2} \mathrm{PE}$ mixture $(2 / 1, n / n)$ in $\mathrm{D}_{2} \mathrm{O}(c=30 \mathrm{mM})$ presenting the amide I region of the infrared spectra during heating (A) and cooling scans (B). The curves are shifted vertically for clarity. 


\section{SUPPORTING INFORMATION}
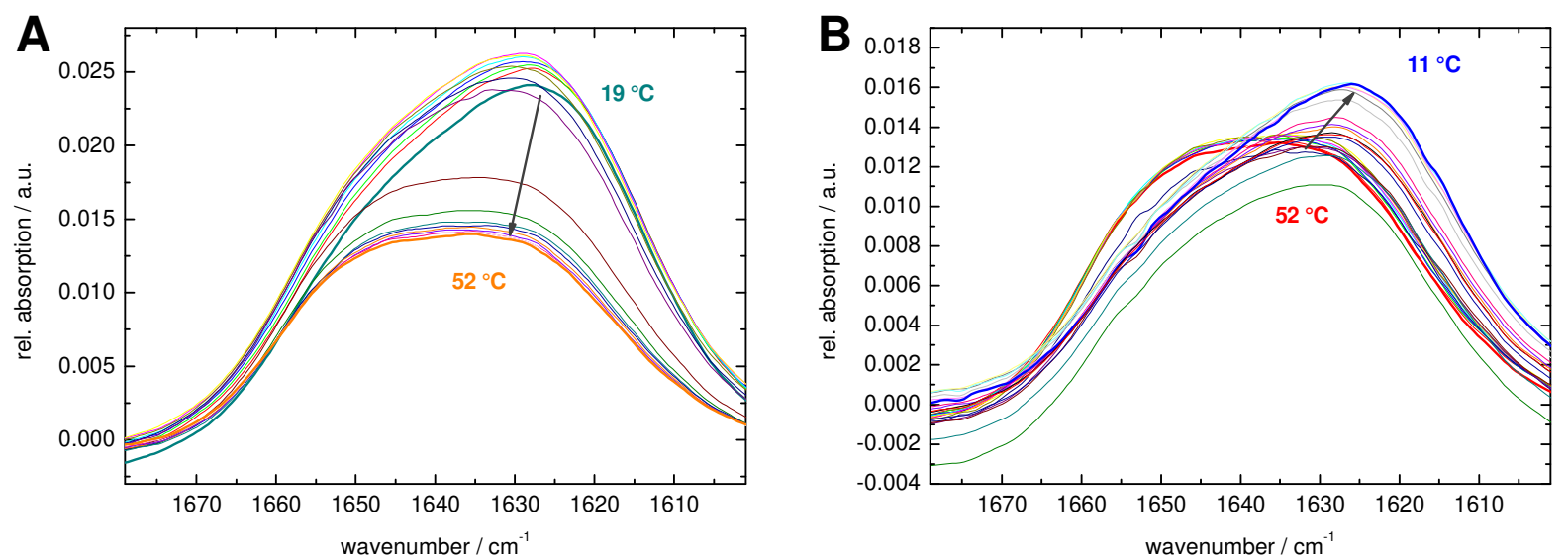

Figure S5. ATR-FTIR measurements of a pure Pad-PC-Pad suspension in $\mathrm{D}_{2} \mathrm{O}(c=30 \mathrm{mM})$ presenting the amide I region of the infrared spectra during heating (A) and cooling scans (B). 